Rev. Elev. Méd. Vét. Pays trop., 1976, 29 (2) : 141-148.

\title{
Description de Rhipicephalus bergeoni n. sp. (Acariens, Ixodida) des montagnes d'Ethiopie
}

\author{
par P. C. MOREL $\left(^{*}\right)$ et J. BALIS $(* *)$
}

\begin{abstract}
RÉSUMÉ
Rhipicephalus bergeon $n$. sp., dont les adultes sont parasites du bétail dans les communautés forestières de montagne et dans les zones d'altitude des prairies subtropicales d'Ethiopie, appartient au groupe de $R h$. sanguineus, quoique certaines particularités morphologiques puissent le faire confondre avec $R h$, appendiculatus, qui semble à ce jour absent de ce pays.
\end{abstract}

Des séries plus ou moins abondantes d'un Rhipicephalus, qui ne correspond à aucune espèce décrite, ont été récoltées en altitude, principalement dans le Chercher. Certaines caractéristiques de sa morphologie se rapprochent de celles de $R h$. appendiculatus Neumann, 1901, de Rh. supertritus Neumann, 1907 et $R h$. turanicus Pomerancev et Matikashvili, 1940; ceci explique qu'il ait pu être confondu avec ces espèces et cité sous ces noms dans la publication de BERGEON et BALIS (1974). Une observation attentive montre que ses affinités le placent à côté de $R h$. sulcatus Neumann, 1908.

\section{DESCRIPTION}

Le type mâle et l'allotype femelle proviennent d'Hubeta (Chercher, Harrar, 24.06.74).

\section{Mâle (fig. 1)}

Basis capituli plus de deux fois plus large que longue; angle de l'auricule droit, au niveau du tiers antérieur de la longueur de la basis ; cornes

(*) I. E. M. V. T., 10, rue Pierre-Curie, 94700 MassonsAlfort, France.

(**) Laboratoire vétérinaire, P. O. Box 19, Debré Zeit, Ethiopie. basidorsales moyennement saillantes; quelques soies (2-4) sur le bord antérieur de l'auricule.

Conscutum à fosses peu profondes, de tégument chagriné, la médiane en ovale allongé, les paramédianes en virgules; sillon scapulaire inapparent, marqué par un alignement de ponctuations pilifères; sillon marginal bien formé, longé intérieurement de petites ponctuations pilifères, délimitant les deux premiers festons ; séries paramédianes et paramarginales de ponctuations pilifères de grande taille; ponctuations porales interstitielles de taille moyenne, peu enfoncées, à bord postérieur non marqué sur la surface du conscutum; tégument du fond des ponctuations pilifères et interstitielles légèrement chagriné, ainsi que sur le fond des sillons marginaux et sur la partie antérieure des fosses scapulo-cervicales.

Feston médian pouvant faire saillie sous forme de mamelon, muni d'un sclérite basal dorsal mais sans sclérite terminal en croissant; épines des coxae du type de celles de $R h$. sanguineus; coxa I sans saillie antérieure dépassant nettement l'angle scapulaire du conscutum en vue dorsale; articles fémoraux, génuaux et tibiaux des pattes III et IV modérément élargis ; plaques adanales en battoirs, à angle interne marqué, antérieur au niveau de l'angle externe arrondi ; stigmates à portion caudale large, à cadre nettement élargi sur son bord dorsal. 

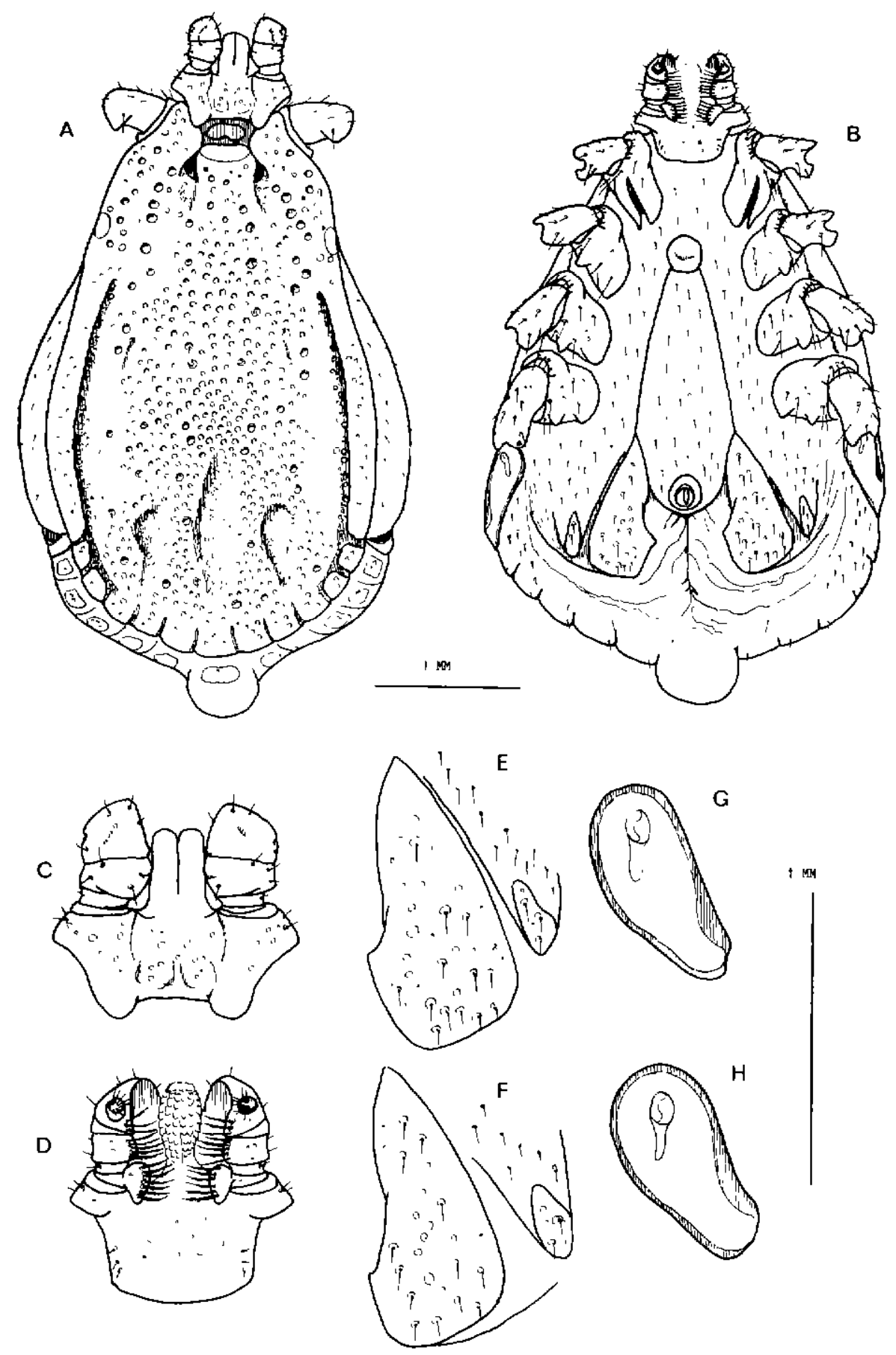

Fig. 1. - Rhipicephalus bergeoni, mâle (exemplaires d'Hubeta) A-F : type ; G-H : paratype. 

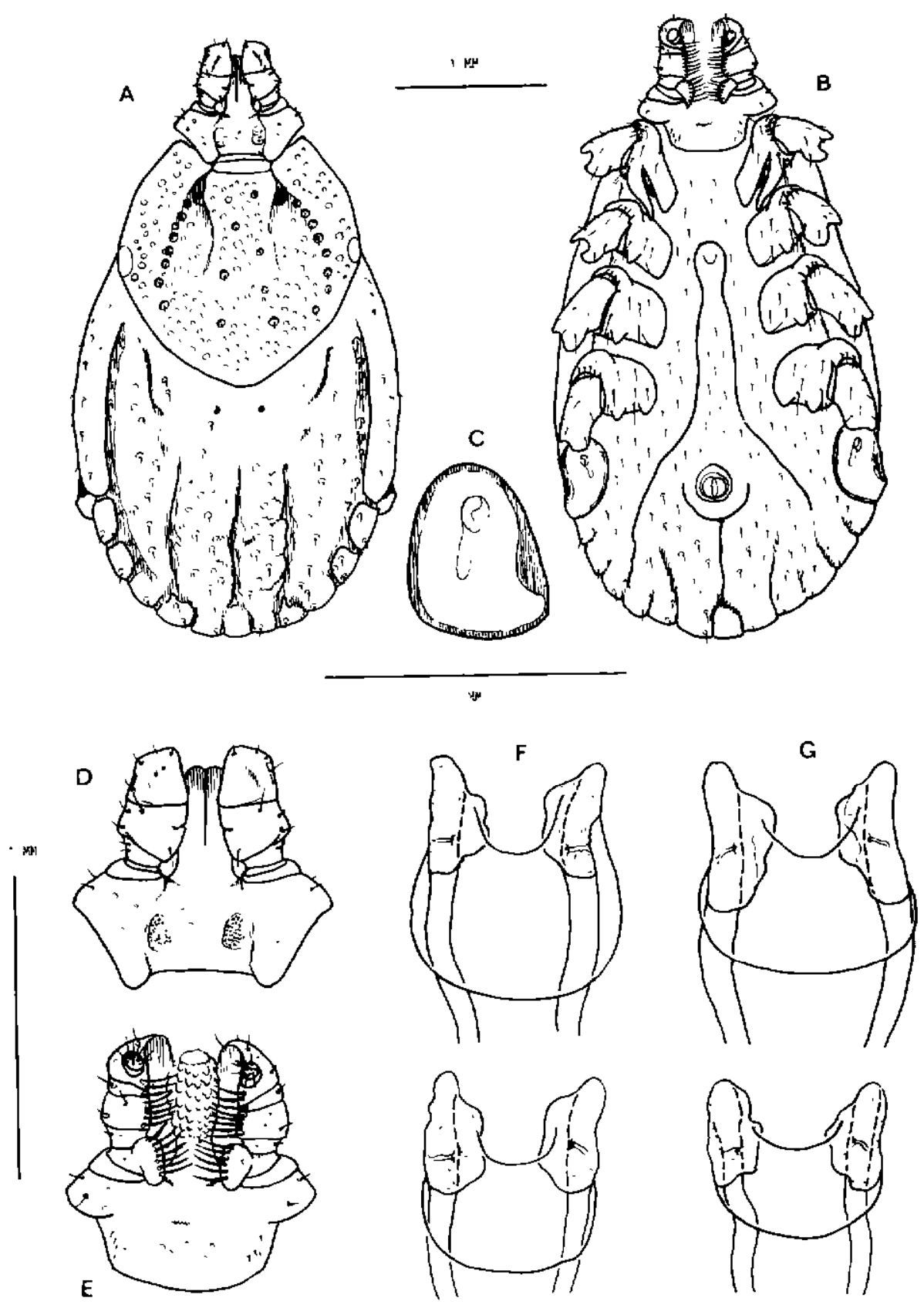

H

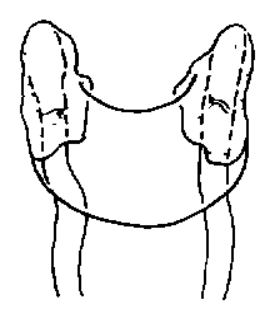

1

Fig. 2. - Rhipicephalus bergeoni, femelle (exemplaires d'Hubeta) A-F : allotype ; G-H : paratypes I : gonopore d'une femelle de Zegeta. 


\section{Femelle (fig. 2)}

Basis capituli plus de deux fois plus large que longue; angle de l'auricule droit, au niveau du tiers antérieur de la basis ; quelques soies (2-4) sur le bord antérieur de l'auricule ; aires poreuses petites, ovalaires, distantes.

Scutum plus long que large, à sillon scapulaire marqué seulement à sa partie antérieure, ou non marqué, indiqué par une série de ponctuations pilifères de grande taille; ponctuations porales interstitielles de taille moyenne ou petite, peu enfoncées, à bord postérieur non marqué sur la surface du scutum; tégument légèrement chagriné sur la partie antérieure des fosses capulocervicales et dans les ponctuations. Alloscutum à sillons marginaux, à fosses médiane et paramédianes marquées, à ponctuations pilifères pourvu de soies courtes, cylindriques ou effilées.

Coxae et épines coxales du type de celles de Rh. sanguineus; sclérites de l'atrium gonoporal larges, débordant intérieurement la lèvre du gonopore par leur expansion ventrale, pigmentés.

\section{COMMENTAIRES}

Les ressemblances entre $R h$. bergeoni et $R h$. appendiculatus consistent dans la présence de tégument chagriné, à des degrés divers d'extension, dans les fosses, sillons et ponctuations, et dans le faible enfoncement des ponctuations interstitielles, chez les deux sexes. Par tous les autres caractères, ces espèces diffèrent et ces divergences coïncident avec les définitions des deux groupes de Rhipicephalus, celui de $R h$. sanguineus et celui de $R h$. appendiculatus. Les caractéristiques différentielles de ce dernier seront donc rappelées ici.

Mâle de $\boldsymbol{R} \boldsymbol{h}$. appendiculatus (fig. 3, exemplaire de Bujumbura, Burundi)

Basis capituli moins de deux fois plus large que longue, à angles des auricules obtus, au niveau du quart antérieur de la basis; séries d'une dizaine de soies sur les faces latérales de la basis, partant des cornes basiventrales, passant sur le bord antérieur des auricules, pour se poursuivre obliquement vers l'arrière sur les faces dorso-latérales de la basis, jusqu'au niveau du milieu de sa longueur; quelques soies sur la face dorso-centrale de la basis prolongent ces séries et les réunissent; cornes basi-dorsales saillantes.
Conscutum à fosses médiane et paramédianes allongées, peu larges et peu profondes, se poursuivant plus ou moins nettement jusque dans les sillons séparant les festons; sillon scapulaire peu marqué ; sillon marginal marqué, à grosses ponctuations pilifères, délimitant un seul feston ; tégument chagriné remplissant complètement les fosses scapulo-cervicales, médiane et paramédianes, les sillons marginaux et les ponctuations; ponctuations pilifères grandes; ponctuations porales interstitielles peu enfoncées.

Feston médian pouvant faire saillie sous forme d'une digitation, pourvu d'un sclérite basal dorsal et d'un sclérite terminal en croissant; plaques adanales approximativement triangulaires, à angle interne du hile au même niveau que l'angle postéro-externe, tous deux très postérieurs ; plaques accessoires réduites ; coxa I pourvue d'un prolongement antérieur dépassant nettement l'angle scapulaire du conscutum en vue dorsale; articles fémoraux, génuaux et tibiaux des pattes III et IV trapus; stigmates à processus caudal large, à cadre du péritrème peu épaissi sur le bord dorsal.

Femelle de $\boldsymbol{R} \boldsymbol{h}$. appendiculatus (fig. 4, exemplaire du Bujumbura, Burundi)

Basis capituli deux fois plus large que longue, avec des séries de 4-8 soies sur les faces latérodorsales et latéro-ventrales de la basis; aires poreuses ovalaires, petites, distantes.

Scutum plus long que large, à sillon scapulaire mal marqué, surtout indiqué par un alignement de ponctuations pilifères grandes et moyennes; ponctuations pilifères du champ cervical grandes ; ponctuations porales interstitielles moyennes, peu enfoncées; tégument chagnné remplissant la plus grande partie des fosses scapulo-cervicales et des ponctuations.

Sclérites de l'atrium gonoporal minces, convexes, sans expansion ventrale vers la lèvre du gonopore, pigmentés.

Etant donné que $R h$. bergeoni appartient manifestement au groupe de $R h$. sanguineus, il est nécessaire de faire des comparaisons morphologiques aussi précises avec Rh. sulcatus qu'avec $R h$. appendiculatus.

Le mâle de $R h$. bergeoni diffère de celui de Rh. sulcatus par la présence de tégument chagriné dans les fosses médiane et paramédianes, dans la partie antérieure des fosses scapulocervicales, dans les sillons marginaux et les 

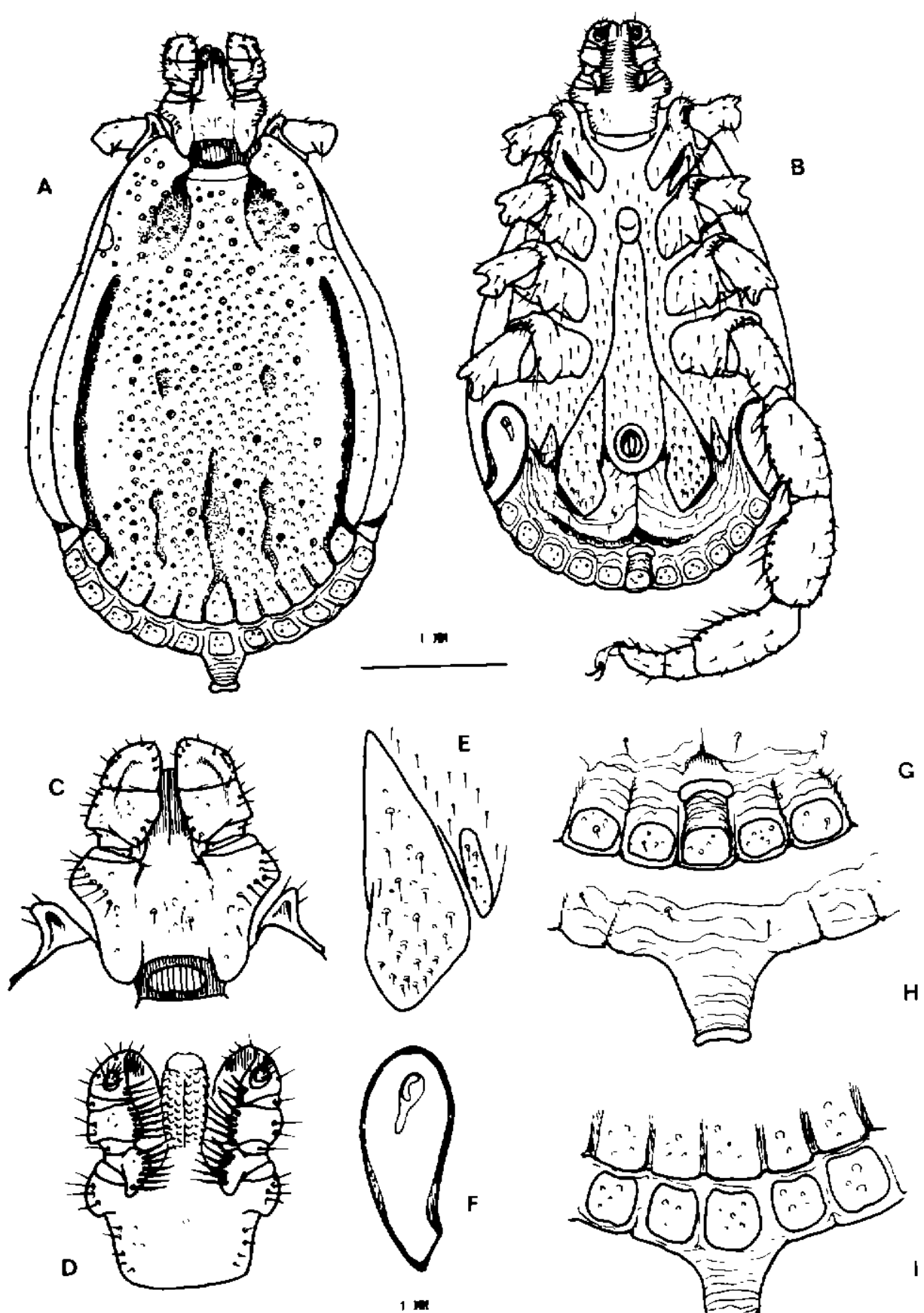

Fig. 3. - Rhipicephalus appendiculatus, mâle (exemplaıres de Bujumbura, Burundi). 

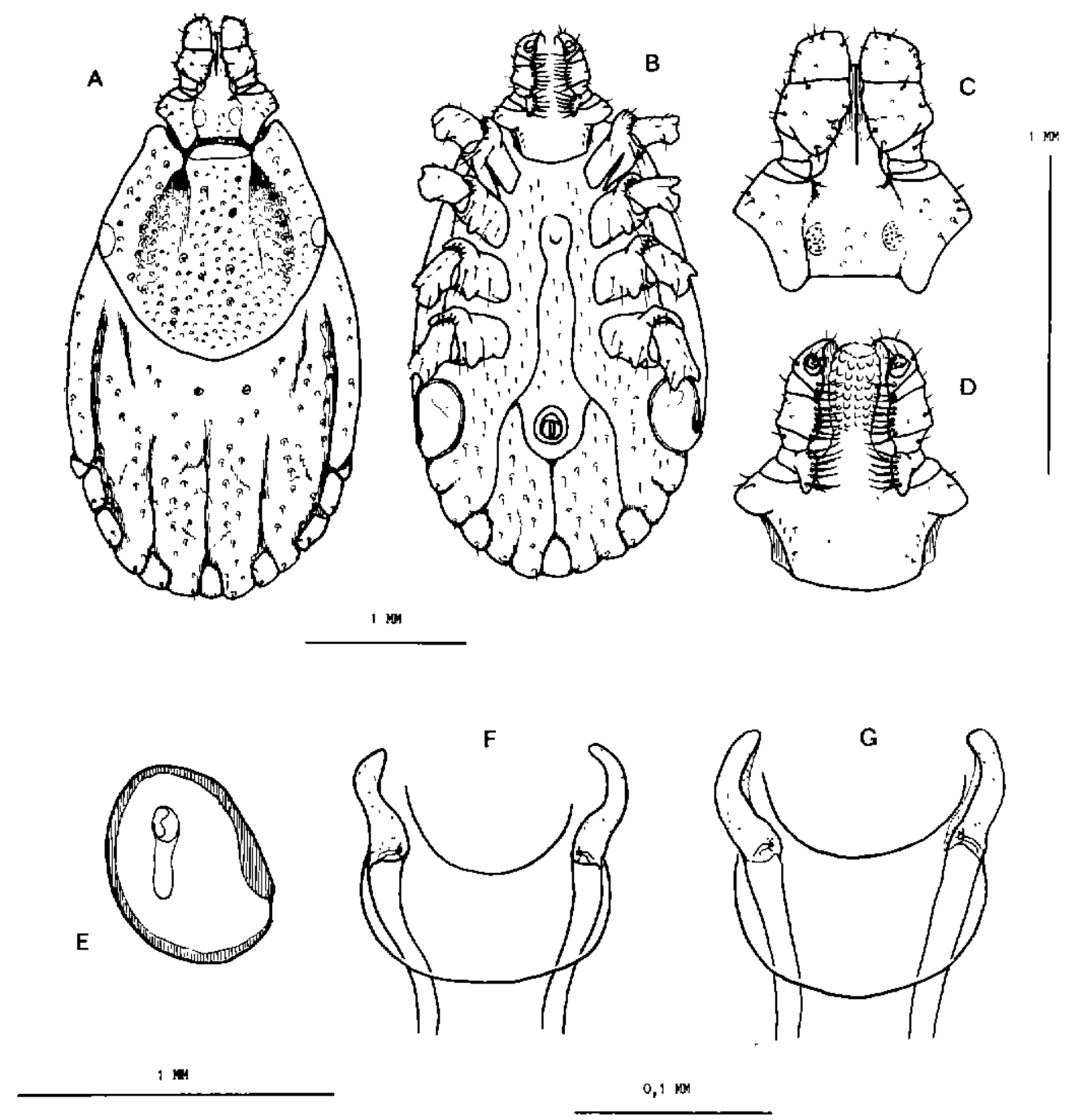

Fig. 4. - Rhipicephalus appendiculatus, femelle (exemplaires de Bujumbura, Burundi).

ponctuations; par le faible enfoncement des ponctuations pilifères et interstitielles (elles sont nettement enfoncées chez $R h$. sulcatus, quelle que soit leur taille).

Les gonopores des femelles de $R h$. bergeoni et de Rh. sulcatus sont analogues. La différenciation des femelles de Rh. bergeoni est basée sur l'effacement des sillons scapulaires (phénomène habituel chez les Rhipicephalus de grande altitude) et sur le faible enfoncement des ponctuations pilifères et interstitielles. Chez $R h$. sulcatus, les sillons scapulaires sont ordinairement bien marqués et les ponctuations pilifères et interstitielles sont enfoncées, quelle que soit leur taille.

La dénomination de la nouvelle espèce a été choisie en hommage confraternel et amical à P. BERGEON, à qui est due la première étude suivie sur les tiques d'Ethiopie.
Nous exprimons des remerciements tout particuliers au Docteur vétérinaire B. LIDOVE, à qui nous sommes redevables de la série importante des $R h$. bergeoni qu'il a récoltés sur les hauteurs du Chercher en juin 1974.

\section{DISTRIBUTION}

\section{Ethiopie}

- BERGEON et BALIS (1974, Rh. appendiculatus p. p.) :

— BAGEMDER ; Gorgora : zébu ( $\mathrm{M}$, $1 \mathrm{~F}, 26 / 08 / 67)$.

- GOJJAM ; Debre Markos (2000 m) (10/67): zébu (49 MM, $24 \mathrm{FF}$ ); mouton : (38 MM, 14 FF).

- BALE; Goba : zébu (28 MM, 05/67). 
- SIDAMO ; Buchisse : zébu (4 MM, 10/69).

- BERGEON et BALIS $(1974, R h$. supertritus) - HARRAR ; Alemaya : Hyaena hyaena (14 MM, 7 FF, 04/67).

- BERGEON et BALIS (1974, Rh. turanicus p.p.) - GOJJAM ; Debre Markos : zébu (85 MM, $8 \mathrm{FF}, 02 / 68)$. SIDAMO; Adola : zébu (20 MM, $11 \mathrm{FF}, 05 / 69)$.

- THEILER (1962, p. 102, Rh. appendiculatus p. p.) - HARRAR ; Alemaya.

- Références nouvelles - BAGEMDER ; Gondar (1 $950 \mathrm{~m})$ : bovin (1 M, 04/67) ; $9 \mathrm{MM}$, 26/08/67) ; Ghenetara (Gondar) : bovin (1 F, 21/11/67).

- BALE; Dodola (2 $500 \mathrm{~m})$ : bovin (15 MM, $11 \mathrm{FF}, 11 / 71)$; Goba (2 $700 \mathrm{~m})$ : bovin (1 M 03/06/74).

- HARRAR ; Debessa (2 $100 \mathrm{~m})$ : bovin (27 MM, 9 FF, 25/06/74) ; Eltoke (2 $200 \mathrm{~m}$ ) : bovin (2 FF, 25/06/74) ; Djara (2 $100 \mathrm{~m})$ : bovin (12 MM, 9 FF, 24/06/74); Alberakate (2 200 m) : bovin (5 MM, 22/06/71); Zegeta (2 $300 \mathrm{~m}$ ) : bovin (17 MM, $5 \mathrm{FF}, 24 / 06 / 74)$; Boroda (2 $300 \mathrm{~m}$ ) : bovin (27 MM, $20 \mathrm{FF}, 25 / 06 / 74)$; Chelenko $(2100 \mathrm{~m})$ : bovin (24 MM, $19 \mathrm{FF}$, 25/06/74) ; Hubeta (2 $500 \mathrm{~m}$ ) : bovin (41 MM, 26 FF, 24/06/74); Horke (2 $300 \mathrm{~m})$ : bovin (35 MM, $21 \mathrm{FF}, 24 / 06 / 74)$.

- SIDAMO ; Adola (1900 m) : bovin (8 MM, $1 \mathrm{~F}, 10 / 71)$; Agare Selam $(2000 \mathrm{~m})$ : bovin $(1 \mathrm{~F}, 28 / 03 / 75)$; Mega $(1900 \mathrm{~m})$ : Hyaena hyaena (12 MM, $25 \mathrm{FF}, 10 / 71)$.

- GEMU GOFA ; Wezo (Chencha) $(2900 \mathrm{~m})$ : bovin (7 MM, $5 \mathrm{FF}, 02 / 12 / 74$ ).

\section{BIOLOGIE}

Du fait de son appartenance au groupe de $R h$. sanguineus, il est vraisemblable que le cycle évolutif de $R h$. bergeoni est triphasique et ditrope, les larves et les nymphes se gorgeant sur les rongeurs myomorphes et les insectivores d'altitude. Il ne pourrait donc en aucune façon être impliqué dans la transmission d'une Theileria.

\section{HABITAT}

Il y a encore trop peu de récoltes de $R h$. bergeoni pour qu'il soit possible de généraliser sa distribution. Il apparaît cependant que l'association dominante de cette espèce intéresse les communautés forestières de montagne, entre 2000 et $3000 \mathrm{~m}$. C'est tout au moins ce qu'il ressort des collections récemment effectuées dans le Chercher. Quelques trouvailles isolées se placent dans les prairies d'altitude, à proximité des communautés forestières.

II est notable que $R h$. bergeoni ne soit pas normalement établi dans les prairies d'altitude tropicales, qui auraient pu constituer l'habitat de $R h$. appendiculatus, en se fondant sur les données d'Afrique orientale équatoriale, s'il avait été présent en Ethiopie. C'est une différence écologique nette entre $R h$. bergeoni et $R h$. appendiculatus.

Les prairies d'altitude d'Ethiopie constituent un hiatus important entre la distribution de $R h$. sulcatus (formations riveraines des steppes masaï, savanes tropicales à fourrés) et celle de $R h$. bergeoni. C'est un argument biologique supplémentaire pour autoriser la distinction entre ces deux espèces, en plus des caractères morphologiques.

Apparemment, $R h$. bergeoni n'existe pas en Afrique équatoriale orientale. Il aurait eu toute chance d'être rencontré au cours des enquêtes récemment publiées sur la Tanzanie et le Kenya, où les tiques caractéristiques des prairies d'altitude et des communautés de montagne sont $R h$. jeanneli, $R h$. bequaerti Zumpt, 1950 et $R h$. hurti Wilson, 1954.

\section{SUMMARY}

Description of Rhipicephalus bergeoni n. sp. (Acarina, Ixodida) of Ethiopia mountains

Rhipicephalus bergeoni $n$. sp., the adults of which are parasites of the cattle in the mountain communities and in the neighbouring subtropical grasslands of Ethiopia, belongs to the Rh. sanguineus group, although on some morphological characters it may be confounded with $R h$. appendiculatus, which seems so far absent from that country. 


\section{RESUMEN}

Descripción de Rhipicephalus bergeoni n. sp. (Acaros, Ixodidae)

de las montañas de Etiopia

Rhipicephalus bergeoni $n$. sp., cuyos adultos son parásitos del ganado en las comunidades forestales de montaña y en las zonas de altura de las praderas subtropicales d'Etiopía, pertenece al grupo de $R h$. sanguineus, a pesar de que ciertas particularidades morfológicas puedan hacerlo confundir con $R h$. appendiculatus, que hasta el dia parece ausente del pais.

\section{BIBLIOGRAPHIE}

BERGEON (P.) et BALIS (J.). Contrıbution à l'étude de la répartition des tiques en Ethiopie (enquête effectuée de 1965 à 1969). Rev. Elev. Méd. vét. Pays trop., 1974,27 (3) : 285-299.

MOREL (P. C.) et VASSILIADES (G.). Les Rhipicephalus du groupe sanguineus: espèces africaines (Acariens, Ixodidae). Rev, Elev. Méd. vét. Pays trop.,
$1962,15(4): 343-386$.

THEILER (G.). The Ixodoidea parasites of vertebrates in Africa south of the Sahara. Onderstepoort (S. 9958) $1962: 255$ p. (mimeogr).

ZUMPT (F.). Preliminary study to a revision of the genus Rhipicephalus. Koch. Doc. Mocambique, 1950, 60 : 57-123. 